九州大学学術情報リポジトリ

Kyushu University Institutional Repository

\title{
A Two Stage Sequential Design in Response Surface Analysis
}

Shirafuji, Michie Kyushu University

https://doi.org/10.5109/12987

出版情報: 統計数理研究. 8 (3/4), pp. 115-126, 1959-03. Research Association of Statistical Sciences バージョン：

権利関係 : 


\title{
A TWO STAGE SEQUENTIAL DESIGN IN RESPONSE SURFACE ANALYSIS
}

\author{
By \\ Michie ShIRAfuJI
}

(Received Dec. 20. 1958)

$\S 1$. Summary. Let us consider a $k$ dimensional response surface of the second degree involving ${ }_{k+2} C_{k}$ unknown parameters $\beta_{0}, \beta_{1}, \cdots, \beta_{k}, \beta_{12}, \cdots$, $\beta_{k-1 k}, \beta_{11}, \cdots, \beta_{k-1 k-1}$ and $\beta_{k k}$ such that

$$
\varphi\left(x_{1}, x_{2}, \cdots, x_{k}\right)=\beta_{0}+\sum_{i=1}^{k} \beta_{i} x_{i}+\sum_{i<j} \beta_{i j} x_{i} x_{j}+\sum_{i=1}^{k} \beta_{i i} x_{i}^{2},
$$

where $x_{1}, x_{2}, \cdots, x_{k-1}$ and $x_{k}$ are fixed variates. to be

The observed values $y_{\alpha}$ at the point $x_{\alpha}=\left(x_{1 \alpha}, x_{2 \alpha}, \cdots, x_{k \alpha}\right)$ are assumed

$$
\boldsymbol{y}_{\alpha}=\varphi\left(\boldsymbol{x}_{1 \alpha}, \boldsymbol{x}_{2 \alpha}, \cdots, \boldsymbol{x}_{k \alpha}\right)+\varepsilon_{\alpha},
$$

where $\varepsilon_{\alpha}(\alpha=1,2, \cdots, N)$ are the error terms distributed independently in the normal distribution $N\left(0, \sigma^{2}\right)$ with a common unknown variance $\sigma^{2}$. (1.01) can also be expressed as

$$
\varphi\left(x_{1}, x_{2}, \cdots, x_{k}\right)=\eta_{0}+\sum_{i=1}^{k} \beta_{i} x_{i}+\sum_{i<j} \beta_{i j} x_{i} x_{j}+\sum_{i=1}^{k} \beta_{i i}\left(x_{i}{ }^{2}-\bar{x}_{i .}{ }^{2}\right),
$$

where we have put in reference with (1.01)

$$
\eta_{0}=\beta_{0}+\sum_{i=1}^{k} \beta_{i i} \overline{x_{i}^{2}},
$$

and

$$
\overline{x_{i \cdot}^{2}}=\frac{1}{N} \sum_{\alpha=1}^{N} x_{i \alpha}^{2}
$$

The design matrix $D_{N, k}$ consists of the $k$ factors and the $N$ experimental points such that

$$
D_{N, k}=\left(\begin{array}{cccc}
x_{11} & x_{21} & \cdots & x_{k 1} \\
x_{12} & x_{22} & \cdots & x_{k 2} \\
\vdots & \vdots & & \vdots \\
x_{1 N} & x_{2 N} & \cdots & x_{k N}
\end{array}\right) .
$$

Our problem is to compare the estimate of $\varphi\left(x_{1}, x_{2}, \cdots, x_{k}\right)$ obtained by the two stage sequential design with the one obtained by the one stage 
designs, under the $k$ dimensional model (1.02) of the second degree. By the two stage sequential design we mean the following procedure. First we make $2^{k}$ experiments with the design matrix $D_{2^{k, k}}$ and decide whether or not we should carry out further experiments on the basis of the outcome of the experiment in the first step. If we decide to make further experiments, we make $(2 k+1)$ additional experiments with the design matrix $D_{2^{k}+2 k+1, k}$ in total. In this paper we shall consider the procedure in the case when $D_{\mathrm{I}^{k}, k}$ is an orthogonal design matrix of two levels and $D_{2^{k}+2 k+1, k}$ is an orthogonal design matrix called as the composite type introduced by G. E. P. Box and K. B. Wilson [3] .

Sequential approaches have been advocated by G. E. P. Box and J. S. Hunter [2] in their response surface analysis, and the object of this paper is to give one of the possible formulations to sequential approach by which to yield an experimenter a basis of a criterion to decide how far sequential approach may be useful. Consequently our paper has the same purpose as T. Kitagawa [6], but, under our special cases and our procedure different from those formulated by him, the results are obtained which are more suited for numerical analysis than the general results given by $T$. Kitagawa [6].

In conclusion the auther wishes her hearty thanks to Prof. T. Kitagawa for his kind encouragement.

2. Least square results. The least square estimates $b_{0}^{(1)}, b_{1}^{(1)}, \cdots, b_{k}^{(1)}$, $b_{12}^{(1)}, \cdots, b_{k-1 k}^{(1)}$ of the unknown parameters $\eta_{01}, \beta_{1}, \cdots, \beta_{k}, \beta_{12}, \cdots, \beta_{k-k k}$ respectively obtained by the experiments with the design matrix $D_{2 k, k}$ are given by the following

$$
\begin{aligned}
& b_{0}^{(1)}=\frac{1}{2^{k}} \sum_{\alpha=1}^{2^{k}} y_{\alpha}, \\
& b_{i}^{(1)}=\frac{1}{2^{k}} \sum_{\alpha=1}^{2^{k}} x_{i \alpha} y_{\alpha} \quad(1 \leqq i \leqq k)
\end{aligned}
$$

and

$$
b_{i j}^{(1)}=\frac{1}{2^{k}} \sum_{\alpha=1}^{2^{k}} \boldsymbol{x}_{i \alpha} \boldsymbol{x}_{j \alpha} \boldsymbol{y}_{\alpha} \quad(1 \leqq i<j \leqq k),
$$

where

$$
\eta_{01}=\beta_{0}+\sum_{i=1}^{k} \beta_{i i} \frac{1}{2^{k}} \sum_{\alpha=1}^{2^{k}} x_{i \alpha}^{2} .
$$

Since $D_{2^{k, k}}$ is an orthogonal design matrix, the unbiased estimates $b_{0}^{(1)}$, $b_{1}^{(1)}, \cdots, b_{k}^{(1)}, b_{12}^{(1)}, \cdots, b_{k-2 i-1}^{(1)}$ and $b_{k-1 k}^{(1)}$ can be easily seen to be normally independently destributed with the common variance $\sigma^{2} / 2^{k}$.

After making the experiments of the second step, the unbiased estimates of all the ${ }_{k+2} C_{k}$ unknown parameters are available which are given by 


$$
\begin{array}{ll}
b_{0}^{(12)}=\frac{1}{2^{k}+2 k+1} \sum_{\alpha=1}^{2^{k}+2 k+1} y_{\alpha}, & \\
b_{i}^{(12)}=\frac{1}{2^{k}+2 \gamma^{2}} \sum_{\alpha=1}^{2^{k}+2 k+1} x_{i \alpha} y_{\alpha} & (1 \leqq i \leqq k), \\
b_{i j}^{(12)}=b_{i j}^{(1)} & (1 \leqq i<j \leqq k)
\end{array}
$$

and

$$
b_{i i}^{(12)}=\frac{1}{B} \sum_{\alpha=1}^{2^{k}+2 k+1}\left(x_{i \alpha}^{2}-\overline{x_{i}^{2}}\right) y_{\alpha} \quad(1 \leqq i \leqq k),
$$

where

$$
\begin{aligned}
& 2 r^{2}=2^{\frac{k}{2}}\left\{\left(2^{k}+2 k+1\right)^{\frac{1}{2}}-2^{\frac{k}{2}}\right\}, \\
& B=2^{k}(1-C)^{2}+2\left(r^{2}-C\right)^{2}+(2 k-1) C^{2}, \\
& C=\left(2^{k}+2 r^{2}\right) /\left(2^{k}+2 k+1\right)
\end{aligned}
$$

and

$$
E\left(b_{0}^{(12)}\right)=\eta_{02}=\beta_{0}+\sum_{i=1}^{k} \beta_{i i} \frac{1}{2^{k}+2 k+1} \sum_{a=1}^{2^{k}+2 k+1} x_{i \alpha}^{2} .
$$

As the design matrix $D_{2^{k}+2 k+1, k}$ is orthogonal, these unbiased estimates are all normally independently distributed. In this case, however, they do have not a common variance. The variances of $b_{0}^{(12)},\left\{b_{i}^{(12)}\right\},\left\{b_{i j}^{(12)}\right\}$ and $\left\{b_{i i}^{(12)}\right\}$ are given by $\left\{\sigma^{2} /\left(2^{k}+2 k+1\right)\right\},\left\{\sigma^{2} /\left(2^{k}+2 \gamma^{2}\right)\right\},\left\{\sigma^{2} / 2^{k}\right\}$ and $\left\{\sigma^{2} / B\right\}$ respectively.

$\S 3$. Two stage procedure. First we define our statistical procedure. $\left(1^{\circ}\right)$ Make experiments involving $2^{k}$ observations $\left\{\boldsymbol{y}_{\alpha}\right\}\left(\alpha=1,2, \cdots, 2^{k}\right)$ according to the design matrix $D_{2 k, k}$.

(2) Calculate the statistic $F^{\prime}$ which is defined by

$$
F^{\prime}=\frac{\nu}{\mu} \frac{2^{k} \sum_{i>i} b_{i j}^{(1) 2}}{2^{k} \hat{\sigma}_{(1)}^{2}},
$$

where $b_{0}^{(1)},\left\{b_{i}^{(1)}\right\}$ and $\left\{b_{i j}^{(1)}\right\}$ are the estimates of $\eta_{i 1},\left\{\beta_{i}\right\}$ and $\left\{\beta_{i j}\right\}$ given by (2.01), (2.02) and (2.03) respectively and

$$
\begin{aligned}
& \hat{\sigma}_{(1)}^{2}=\frac{1}{2^{k}} \sum_{\alpha=1}^{2^{k}}\left(y_{\alpha}-b_{0}^{(1)}-\sum_{i=1}^{k} b_{i}^{(1)} x_{i \alpha}-\sum_{i<j} b_{i j}^{(1)} x_{i \alpha} x_{j \alpha}\right)^{2}, \\
& \nu=2^{k}-\left(k^{2}+k+2\right) / 2
\end{aligned}
$$

and

$$
\mu=k(k-1) / 2 \text {. }
$$

Since $\hat{\sigma}_{(1)}^{2}$ is independent to $b_{0}^{(1)},\left\{b_{i}^{(1)}\right\}$ and $\left\{b_{i j}^{(1)}\right\}, F^{\prime}$ is distributed accord- 
ing to the non-central $F$-distribution with the pair of the degrees of free$\operatorname{dom}(\mu, \nu)$ and the non-centricity parameter

$$
\lambda=2^{k} \sum_{i<j} \beta_{i j}^{2} / 2 \sigma^{2} .
$$

Now let $\alpha$ be an assigned level of significance, such that $0<\alpha<1$. $\left(3^{\circ}\right)_{1}$ If $F^{\prime}$ turns out to be significantly large, that is,

$$
F^{\prime} \geqq F_{\nu}^{\mu}(\alpha),
$$

which is the $\alpha$-point of the $F$-distribution with the pair of the degrees of freedom $(\mu, \nu)$, calculate

$$
\hat{\varphi}_{1}\left(x_{1}, x_{2}, \cdots, x_{k}\right)=b_{0}^{(1)}+\sum_{i=1}^{k} b_{i}^{(1)} x_{i}+\sum_{i<j} b_{i j}^{(1)} x_{i} x_{j}
$$

as the estimate of $\varphi\left(x_{1}, x_{2}, \cdots, x_{k}\right)$.

$\left(3^{\circ}\right)_{2}$ In the contary case, that is, when

$$
F^{\prime}<F_{\nu}^{\mu}(\alpha),
$$

we make experiments of the second step according to the design matrix $D_{2^{k}+1, k}$ given by

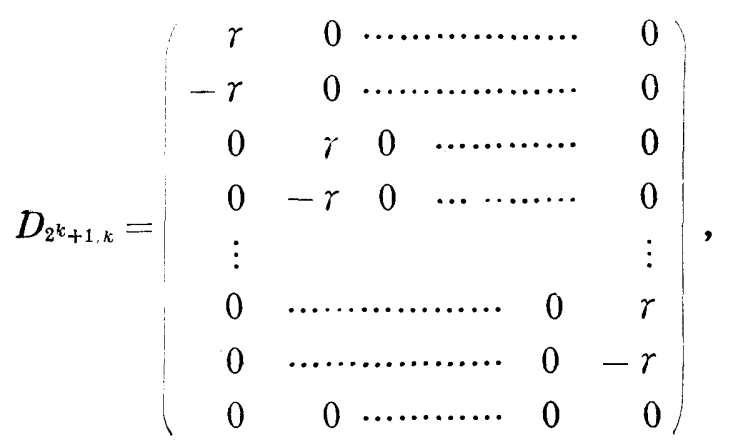

where $r$ is given by (2.05). And then calculate

$$
\begin{aligned}
& \hat{\varphi}_{2}\left(x_{1}, x_{2}, \cdots, x_{k}\right) \\
= & b_{0}^{(12)}+\sum_{i=1}^{k} b_{i}^{(12)} x_{i}+\sum_{i>1} b_{i j}^{(12)} x_{i} x_{j}+\sum_{i=1}^{k} b_{i i}^{(12)}\left(x_{i}^{2}-\overline{x_{i .}^{2}}\right),
\end{aligned}
$$

for the estimate of $\varphi\left(x_{1}, x_{2}, \cdots, x_{k}\right)$.

We are proposing this estimate after the following motive. If the sum of squares of the interaction terms turns out to be significantly large compared with the error term, the model of the response surface which takes the first three terms of (1.03) into account would be a valid approximation to the true response surface, and we may be satisfied by the estimate of the response surface obtained from the experiments which yield us the estimates under the assumption that the fourth term in (1.03) is equal to zero. On the other hand, if it does not turn out to be significantly large compared with the error term, it will not be a valid approximation unless we do not 
take the fourth term in (1.03) into account. To avail the estimate which satisfies such requirements we need to make further experiments. In short we are dealing with the statistical procedure applied to an incompletely specified model, that is, estimation after preliminary test of significance formulated and developed by several authers such as T. A. Bancroft [1], T. Kitagawa [4] and A. E. Paul [7].

4. Main results. It is evident that $\hat{\varphi}\left(x_{1}, \cdots, x_{k}\right)$ is not generally an unbiased estimate of $\varphi\left(x_{1}, \cdots, x_{k}\right)$. To see the nature of our estimate discussed in $\S 3$, we may therefore appeal to the mean square error

$$
E\left\{\hat{\varphi}\left(x_{1}, \cdots, x_{k}\right)-\varphi\left(x_{1}, \cdots, x_{k}\right)^{2}\right\}
$$

for each assigned point $x=\left(x_{1}, \cdots, x_{k}\right)$ in the $k$-dimensional space. It will be, however, interesting to consider the norm defined by

$$
E\left\{\int_{D} \ldots \int\left|\hat{\varphi}\left(x_{1}, \cdots, x_{k}\right)-\varphi\left(x_{1}, \cdots, x_{k}\right)\right|^{2} d x_{1} \cdots d x_{k}\right\},
$$

where $\mathfrak{D}$ is the domain in our consideration in the $k$-dimensional euclidian space.

As our problem is to discuss the merits of estimate obtained through the two stage design, we need, in addition to the norm of $\hat{\varphi}\left(x_{1}, \cdots, x_{k}\right)$, to calculate the estimate $\hat{\varphi}_{1}\left(x_{1}, \cdots, x_{k}\right), \hat{\varphi}_{2}\left(x_{1}, \cdots, x_{k}\right)$ of $\varphi\left(x_{1}, \cdots, x_{k}\right)$ obtained by the one stage designs $D_{2^{k}, k}$ and $D_{2^{k}+2 k+1, k}$ respectively. The norm depends on the domain of integration $(\mathcal{D}$ and there is a good deal of freedom in the selection of $\mathfrak{D}$. It appears, however, to be most appropriate to take $\mathfrak{D}$ as $k$-dimensional cube which is defined by $\mathfrak{D}_{R}: \sum_{i=1}^{k} x_{i}{ }^{2} \leqq R$.

Hence we need to calculate

$$
\begin{aligned}
& N_{2^{k}}=E\left\{\iint_{D_{R}} \ldots \int\left|\hat{\varphi}_{1}\left(x_{1}, \cdots, x_{k}\right)-\varphi\left(x_{1}, \cdots, x_{k}\right)\right|^{2} d x_{1} \cdots d x_{k}\right\}, \\
& N_{2^{k}+2 k+1}=E\left\{\int_{D_{R}}^{\cdots}|| \hat{\varphi}_{2}\left(x_{1}, \cdots, x_{k}\right)-\left.\varphi\left(x_{1}, \cdots, x_{k}\right)\right|^{2} d x_{1} \cdots d x_{k}\right\}
\end{aligned}
$$

and

$$
\begin{aligned}
& N_{2^{k}+(1-p)(2 k+1)}^{*}=E\left\{\int_{\mathscr{D}_{R}}^{\ldots} \int \hat{\varphi}\left(x_{1}, \cdots, x_{k}\right)-\left.\varphi\left(x_{1}, \cdots, x_{k}\right)\right|^{2} d x_{1} \cdots d x_{k}\right\} \\
& \quad=\operatorname{Pr} .\{L\} E_{L}\left\{\int_{\mathfrak{D}_{R}}^{\ldots} \int\left|\hat{\varphi}_{1}\left(x_{1}, \cdots, x_{k}\right)-\varphi\left(x_{1}, \cdots, x_{k}\right)\right|^{2} d x_{1} \cdots d x_{k}\right\} \\
& \quad+\operatorname{Pr} .\left\{L^{c}\right\} E_{L^{c}}\left\{\int_{D_{R}}^{\ldots} \int\left|\hat{\varphi}_{2}\left(x_{1}, \cdots, x_{k}\right)-\varphi\left(x_{1}, \cdots, x_{k}\right)\right|^{2} d x_{1} \cdots d x_{k}\right\},
\end{aligned}
$$

where $L$ is an event $F^{\prime} \geqq F_{\nu}^{\mu}(\alpha), L^{c}$ is its complement and 


$$
\begin{aligned}
p & =\operatorname{Pr} .\{L\}=\int_{F_{\nu}^{\mu}(\alpha)}^{\infty} g_{\mu, \nu}(F: \lambda) d F \\
& =\sum_{i=0}^{\infty} \frac{e^{-\lambda} \lambda^{i}}{i !}\left\{1-I_{h}\left(\frac{\mu}{2}+i, \frac{\nu}{2}\right)\right\} \\
& =\sum_{i=0}^{\infty} \frac{e^{-\lambda} \lambda^{i}}{i !} I_{1-h}\left(\frac{\nu}{2}, \frac{\mu}{2}+i\right),
\end{aligned}
$$

where $g_{\mu, \nu}(F: \lambda)$ is the probability density of the non-central $F$-distribution with the pair of the degrees of freedom $(\mu, \nu)$ and the non-centricity parameter $\lambda$, where $\mu, \nu, \lambda$ and $h$ are given by (3.03), (3.04) and (3.05),

$$
h=\frac{\mu}{\nu} F_{\nu}^{\mu}(\alpha) /\left(1+\frac{\mu}{\nu} F_{\nu}^{\mu}(\alpha)\right) \text {. }
$$

We have put

$$
I_{h}(m, n)=\frac{B_{h}(m, n)}{B(m, n)},
$$

where

$$
\text { (4.09) } \quad B_{h}(m, n)=\int_{0}^{h} t^{m-1}(1-t)^{n-1} d t, \quad m, n<0, \quad 0<h \leqq 1 .
$$

Theorem 1. We have

$$
N_{2^{k}+(1-p)(2 k+1)}^{*}=p N_{2^{k}}+(1-p) N_{2^{k}+2 k+1},
$$

where

(4.11) $\quad N_{2^{k}}=\frac{R^{\frac{k}{2}} \Pi^{k}}{2^{k} \Gamma\left(\frac{k}{2}+3\right)}\left[\sigma^{2}\left\{\left(\frac{k}{2}+2\right)\left(\frac{k}{2}+1\right) \frac{1}{2^{k}}+R\left(\frac{k}{2}+2\right) \frac{1}{2} \frac{k}{2^{k}}\right.\right.$

$$
\begin{aligned}
& \left.+R^{2} \frac{k(k-1)}{2^{3}} \frac{1}{2^{k}}\right\}+\frac{R^{2}}{2} \sum_{i=1}^{k} \beta_{i i}^{2} \\
& \left.+\left\{\left(\frac{k}{2}+2\right)\left(\frac{k}{2}+1\right)-R\left(\frac{k}{2}+2\right)+R^{2}\right\}\left(\sum_{i=1}^{k} \beta_{i i}\right)^{2}\right],
\end{aligned}
$$

(4.12) $\quad N_{2^{k}+2 k+1}=\frac{R^{k} \Pi_{2}^{k}}{2^{k} \Gamma\left(\frac{k}{2}+3\right)} \sigma^{2}\left[\left(\frac{k}{2}+2\right)\left(\frac{k}{2}+1\right) \frac{1}{2^{k}+2 k+1}\right.$

$$
\begin{aligned}
& +R\left(\frac{k}{2}+2\right) \frac{1}{2} \frac{k}{2^{k}+2 \gamma^{2}}+R^{2} \frac{k(k-1)}{2^{3}} \frac{1}{2^{k}} \\
& \left.+\frac{k}{B}\left\{C^{2}\left(\frac{k}{2}+2\right)\left(\frac{k}{2}+1\right)-R C\left(\frac{k}{2}+2\right)+R^{2} \frac{3}{4}\right\}\right],
\end{aligned}
$$

and $B, C$ and $p$ are given by (2.10), (2.11) and (4.06). 
Proof. Let us note that we have the following formula

$$
\int \ldots \int x_{i}^{m} x_{j}^{n} d x_{1} \cdots d x_{k}=\frac{R^{\frac{m+n+k}{2}} \Gamma\left(\frac{m+1}{2}\right) \Gamma\left(\frac{n+1}{2}\right)}{2^{k}} \frac{\Gamma\left(\frac{m+n+k}{2}+1\right)}{\Gamma}
$$

for $m, n>-1$ and $1 \leqq i, j \leqq k(i \neq j)$.

It follows, in view of $\S 2$, that $b_{0}^{(1)},\left\{b_{i}^{(1)}\right\},\left\{b_{i j}^{(1)}\right\}$ and $\hat{\sigma}_{(1)}^{2}$ are mutually independent and the variances of $b_{0}^{(1)},\left\{b_{i}^{(1)}\right\}$ and $\left\{b_{i j}^{(1)}\right\}$ are equal to the common value $\sigma^{2} / 2^{k}$. Hence in view of $\S 2$ and (4.13), the first term of the right hand side of (4.05) may be written

$$
\begin{aligned}
& \operatorname{Pr} .\{L\} E_{L}\left\{\int_{D_{R}}^{\ldots} \int\left|\hat{\varphi}_{1}\left(x_{1}, \cdots, x_{k}\right)-\varphi\left(x_{1}, \cdots, x_{k}\right)\right|^{2} d x_{1} \cdots d x_{k}\right\} \\
& =p \frac{R^{\frac{k}{2}} \Pi^{\frac{k}{2}}}{2^{k} \Gamma\left(\frac{k}{2}+3\right)}\left[\sigma^{2}\left\{\left(\frac{k}{2}+2\right)\left(\frac{k}{2}+1\right) \frac{1}{2^{k}}+R\left(\frac{k}{2}+2\right) \frac{1}{2} \frac{k}{2^{k}}\right\} .\right. \\
& \left.+\frac{R^{2}}{2} \sum_{i=1}^{k} \beta_{i i}^{2}+\left\{\left(\frac{k}{2}+2\right)\left(\frac{k}{2}+1\right)-R\left(\frac{k}{2}+2\right)+\frac{R^{2}}{4}\right\}\left(\sum_{i=1}^{k} \beta_{i i}^{2}\right)^{2}\right] \\
& +\frac{R^{\frac{k}{2}+2}}{2^{k}} \frac{\left\{\Gamma\left(\frac{3}{2}\right)\right\}^{2} \Pi^{\frac{k-2}{2}}}{\Gamma\left(\frac{k}{2}+3\right)} \operatorname{Pr} .\left\{L \left\{E_{L}\left\{\sum_{i<j}\left(b_{i j}^{(1)}-\beta_{i j}\right)^{2}\right\} .\right.\right.
\end{aligned}
$$

On the other hand, it follows that $b_{0}^{(12)},\left\{b_{i}^{(12)}\right\},\left\{b_{i j}^{(12)}\right\}$ and $\left\{b_{i i}^{(12)}\right\}$ are mutually independent in virtue of $\S 2$. Furthermore we have, in view of (2.15), (4.16), (4.17) and (4.18), $b_{0}^{(12)},\left\{b_{i}^{(12)}\right\},\left\{b_{i j}^{(12)}\right\}$ and $\left\{b_{i \imath}^{(12)}\right\}$ are independ. ent to $\hat{\sigma}_{(1)}^{\hat{2}}$. Since $b_{0}^{(1)}$ and $\sum_{\alpha=2^{k}+1}^{2 k+2 k+1} y_{\alpha}$ are independent to $\hat{\sigma}_{(1)}^{2}$,

$$
b_{0}^{(12}=\left(2^{k} b_{0}^{(1)}+\sum_{a=2^{k}+1}^{2^{k}+2 k+1} y_{a}\right) /\left(2^{k}+2 k+1\right)
$$

is independent to $\hat{\sigma}_{(1)}^{2}$. Similarily we have that

$$
\begin{aligned}
& b_{i}^{(12)}=\left(2^{k} b_{i}^{(1)}+\sum_{\alpha=2^{k}+1}^{2^{k}+2 k+1} x_{i \alpha} y_{\alpha}\right) /\left(2^{k}+2 r^{2}\right) \quad(1 \leqq i \leqq k), \\
& b_{i j}^{(12)}=b_{i j}^{(1)} \quad(1 \leqq i<j \leqq k)
\end{aligned}
$$

and

$$
\begin{aligned}
b_{b i}^{(12)} & =\frac{1}{B} \sum_{\alpha=1}^{2^{k}+2 k+1}\left(x_{i \alpha}^{2}-\overline{x_{i}^{2} .}\right) y_{\alpha} \\
& =\frac{1-C}{B} 2^{k} b_{0}^{(1)}+\frac{1}{B} \sum_{\alpha=2^{k}+1}^{2^{k}+2 k+1}\left(x_{i \alpha}^{2}-\overline{x_{i}^{2}}\right) y_{\alpha} \quad(1 \leqq i \leqq k)
\end{aligned}
$$

are independent to $\hat{\sigma}_{(1)}^{2}$. 
Now the second term of the right hand side of (4.05) may be written (4. 19)

$$
\begin{aligned}
& \operatorname{Pr} .\left\{L^{c}\right\} E_{L^{c}}\left\{\int_{\mathfrak{D}_{R}} \ldots\left\{\hat{\varphi}_{2}\left(x_{1}, \cdots, x_{k}\right)-\left.\varphi\left(x_{1}, \cdots, x_{k}\right)\right|^{2} d x_{1} \cdots d x_{k}\right\}\right. \\
& =(1-p) \frac{R^{\frac{k}{2}} \Pi_{2}^{k}}{2^{k} \Gamma\left(\frac{k}{2}+3\right)} \sigma^{2}\left[\left(\frac{k}{2}+2\right)\left(\frac{k}{2}+1\right) \frac{1}{2^{k}+2 k+1}\right. \\
& +R\left(\frac{k}{2}+2\right) \frac{1}{2} \frac{k}{2^{k}+2 r^{2}} \\
& \left.+\frac{k}{B}\left\{C^{2}\left(\frac{k}{2}+2\right)\left(\frac{k}{2}+1\right)-R C\left(\frac{k}{2}+2\right)+R^{2} \frac{3}{4}\right\}\right] \\
& +\frac{R^{k}{ }^{k+2}\left\{\Gamma\left(\frac{3}{2}\right)\right\}^{2} \Pi^{\frac{k-2}{2}}}{2^{k} \Gamma\left(\frac{k}{2}+3\right)} \operatorname{Pr} .\left\{L^{c}\right\} E_{L^{c}}\left\{\sum_{i<j}\left(b_{i j}^{(12)}-\beta_{i j}\right)^{2}\right\} .
\end{aligned}
$$

Here we have, in view of (4.17) and the variances of $\left\{b_{i j}^{(1)}\right\}$ are given by $\sigma^{2} / 2^{k}$, (4. 20)

$$
\begin{aligned}
& \operatorname{Pr} .\{L\} E_{L} \sum_{i<j}\left(b_{i j}^{(1)}-\beta_{i j}\right)^{2}+\operatorname{Pr} .\left\{L^{c}\right\} E_{L^{c}}\left\{\sum_{i<j}\left(b_{i j}^{(12)}-\beta_{i j}\right)^{2}\right) \\
= & E\left\{\sum_{i<j}\left(b_{i j}^{(1)}-\beta_{i j}\right)^{2}\right\} \\
= & \frac{k(k-1)}{2} \cdot \frac{\sigma^{2}}{2^{k}} .
\end{aligned}
$$

Consequently (4.10) follows from (4.14), (4.15) and (4.20), as has to be proved.

\$. Numerical results. In case $R=1$ and $k=3,4,5,6$, we have that the norms of our estimates (4.10), (4.11), (4.12) divided by the population variance are given by the following
(5.01) $\quad N_{2^{3}}^{\prime}=0.0898\left\{1.8439+0.5 \sum_{i=1}^{3} \beta_{i i}^{2} / \sigma^{2}+5.50\left(\sum_{i=1}^{3} \beta_{i i} / \sigma\right)^{2}\right\} \quad k=3$,
(5.02) $\quad N_{2^{4}}^{\prime}=0.0257\left\{1.3438+0.5 \sum_{i=1}^{4} \beta_{i i}^{2} / \sigma^{2}+8.25\left(\sum_{i=1}^{4} \beta_{i i} / \sigma\right)^{2}\right\} \quad k=4$,
(5.03) $\quad N_{2^{5}}^{\prime}=0.0157\left\{0.9219+0.5 \sum_{i=1}^{5} \beta_{i i}^{2} / \sigma^{2}+11.50\left(\sum_{i=1}^{5} \beta_{i i} / \sigma\right)^{2}\right\} \quad k=5$,
(5.04) $\quad N_{2^{8}}^{\prime}=0.0040\left\{0.6055+0.5 \sum_{i=1}^{6} \beta_{i i}^{2} / \sigma^{2}+15.25\left(\sum_{i=1}^{6} \beta_{i i} / \sigma\right)^{2}\right\} \quad k=6$,
(5.05) $\quad N_{2^{3}+6+1}^{\prime}=0.0898 \times 3.1192=0.2801 \quad k=3$,
(5.06) $\quad N_{2^{\prime}+8+1}^{\prime}=0.0257 \times 3.5888=0.0922 \quad k=4$,
(5. 07) $\quad N_{2^{5}+10+1}^{\prime}=0.0157 \times 3.6691=0.0576 \quad k=5$,
(5.08) $\quad N_{2^{6}+12+1}^{\prime}=0.0040 \times 4.4678=0.0179 \quad k=6$ 
and

$$
N_{k(\alpha)}^{* \prime}=p N_{2^{k}}^{\prime}+(1-p) N_{2^{k}+2 k+1}^{\prime}
$$

where we put

$$
\begin{aligned}
& N_{2^{k}}^{\prime}=N_{2^{k}} / \sigma^{2}, \\
& N_{2^{k}+2 k+1}^{\prime}=N_{2^{k}+2 k+1} / \sigma^{2},
\end{aligned}
$$

and

$$
N_{k(\alpha)}^{* \prime}=N_{2^{k}+(1-p)(2 k+1)}^{*} / \sigma^{2} .
$$

Numerical tabulation of the values $N_{2^{k}}^{\prime}, N_{2^{k}+2 k+1}^{\prime}, N_{k(\alpha)}^{* \prime}$ was carried out assuming

$$
\beta_{i j}=\beta^{*} \quad 1 \leqq i<j \leqq k
$$

and

$$
\beta_{i i}=\beta^{* *} \quad 1 \leqq i \leqq k,
$$

for $\alpha=0.05,0,25, \sigma^{2} / \beta^{*^{2}}=50(50) 200$ and

$$
r=\left(\frac{\beta^{* *}}{\sigma}\right)^{2} /\left(\frac{\beta^{*}}{\sigma}\right)^{2}
$$

$=1(1) 5$ and $r^{-1}=1$ (1) 5 . For the numerical tabulation of $p$ defined by (4. 06), we made use of the incomplete beta-function by $K$. Pearson [8] and Tables of the Poisson distribution by T. Kitagawa [5].

KyUSHU UNIVERSITy 
Table 1. Values of $N^{\prime} 2 k, N^{* *}{ }_{k(\alpha)}$ and $N^{\prime}{ }_{2 k+2 k+1}$ when $\sigma^{2} / \beta^{* 2}=50$.

\begin{tabular}{|c|c|c|c|c|c|c|c|c|c|}
\hline$\sigma_{\sigma^{-2} \text { (Norm) }}^{r}-r$ & 5 & 4 & 3 & 2 & 1 & $\frac{1}{2}$ & $\frac{1}{3}$ & $\frac{1}{4}$ & $\frac{1}{5}$ \\
\hline$N_{23}^{\prime}$ & 0.6236 & 0.5320 & 0.4404 & 0.3488 & 0.2572 & 0.2114 & 0.1961 & 0.1885 & 0.1839 \\
\hline$N^{* \prime}{ }_{3(0.25)}$ & 0.3721 & 0.3476 & 0.3231 & 0.2985 & 0.2740 & 0.2617 & 0.2576 & 0.2556 & 0.2543 \\
\hline$N^{* \prime}{ }_{3(0.05)}$ & 0.2872 & 0.2853 & 0.2834 & 0.2815 & 0.2796 & 0.2787 & 0.2784 & 0.2782 & 0.2781 \\
\hline$N_{2}^{\prime}+6+1$ & \multicolumn{9}{|l|}{0.2801} \\
\hline$N_{2}^{\prime}{ }_{24}$ & 0.3789 & 0.3100 & 0.2412 & 0.1723 & 0.1034 & 0.0690 & 0.0575 & 0.0518 & 0.0483 \\
\hline$N^{* \prime}{ }_{4(0.25)}$ & 0.1940 & 0.1695 & 0.1451 & 0.1206 & 0.0962 & 0.0840 & 0.0799 & 0.0779 & C.0766 \\
\hline$N^{* \prime}{ }_{4(0.05)}$ & 0.1162 & 0.1104 & 0.1047 & 0.0989 & 0.0932 & 0.0903 & 0.0893 & 0.0889 & 0.0886 \\
\hline$N^{\prime} 2^{4}+8+1$ & \multicolumn{9}{|l|}{0.0922} \\
\hline$N^{\prime} 2^{5}$ & $0.4 \overline{6} 98$ & 0.3787 & 0.2877 & 0.1966 & 0.1055 & 0.0500 & 0.0448 & 0.0372 & 0.0327 \\
\hline$N^{* \prime}{ }_{5(0.25)}$ & 0.2987 & 0.2454 & 0.1922 & 0.1389 & 0.0856 & 0.0590 & 0.0501 & 0.0457 & 0.0430 \\
\hline$N^{* \prime \prime}(0.05)$ & 0.1466 & 0.1270 & 01073 & 0.0876 & 0.0680 & 0.0581 & 0.0548 & 0.0532 & 0.0522 \\
\hline$N^{\prime} 2^{5}+10+1$ & \multicolumn{9}{|l|}{0.0576} \\
\hline$N_{26}^{\prime}$ & 0.2232 & 0.1791 & 0.1349 & 0.0907 & 0.0466 & 0.0245 & 0.0171 & 0.0135 & 0.0113 \\
\hline$N_{6(0.25)}^{* \prime}$ & 0.2068 & 0.1662 & 0.1255 & 0.0849 & 0.0443 & 0.0240 & 0.0172 & 0.0138 & 0.0118 \\
\hline$N_{6(0.05)}^{* \prime}$ & 0.1528 & 0.1238 & 0.0948 & 0.0657 & 0.0367 & 0.0222 & 0.0174 & 0.0150 & 0.0135 \\
\hline$N^{\prime} 26+12+1$ & \multicolumn{9}{|l|}{0.0179} \\
\hline
\end{tabular}

Table 2. Values of $N^{\prime} 2 k, N^{* \prime}{ }_{k(\alpha)}$ and $N^{\prime}{ }^{k}+2 k+1$ when $\sigma^{2} / \beta^{* 2}=100$.

\begin{tabular}{|c|c|c|c|c|c|c|c|c|c|}
\hline$\sigma_{\sigma^{-2}(\text { Norm })}^{r}$ & 5 & 4 & 3 & 2 & 1 & $\frac{1}{2}$ & $\frac{1}{3}$ & $\begin{array}{l}1 \\
4\end{array}$ & $\begin{array}{l}1 \\
5\end{array}$ \\
\hline$N_{23}^{\prime}$ & 0.3946 & 0.3488 & 0.3030 & 0.2572 & 0.2114 & 0.1885 & 0.1808 & 0.1770 & 0.1747 \\
\hline$N_{3(0.25)}^{* \prime}$ & 0.3097 & 0.2979 & 0.2860 & 0.2742 & 0.2623 & 0.2564 & 0.2544 & 0.2534 & 0.2529 \\
\hline$N^{* \prime}{ }_{3(0.05)}$ & 0.2824 & 0.2815 & 0.2806 & 0.2797 & 0.2787 & 0.2783 & 0.2781 & 0.2781 & 0.2780 \\
\hline$N^{\prime} 2^{3}+8+1$ & \multicolumn{9}{|l|}{0.2801} \\
\hline$N_{24}^{\prime}$ & 0.2067 & 0.1723 & 0.1378 & 0.1034 & 0.0690 & 0.0518 & 0.0460 & 0.0431 & 0.0414 \\
\hline$N^{* \prime}{ }_{4(0.25)}$ & 0.1269 & 0.1165 & 0.1060 & 0.0956 & 0.0852 & 0.0800 & 0.0782 & 0.0774 & 0.0768 \\
\hline$N^{* \prime}{ }_{4(0.05)}$ & 0.0998 & 0.0975 & 0.0952 & 0.0930 & 0.0907 & 0.0896 & 0.0892 & 0.0890 & 0.0889 \\
\hline$N^{\prime} 2^{4}+8+1$ & \multicolumn{9}{|l|}{0.0922} \\
\hline$N^{\prime} 2^{5}$ & 0.2421 & 0.1966 & 0.1511 & 0.1055 & 0.0600 & 0.0372 & 0.0296 & 0.0259 & 0.0236 \\
\hline$N^{* \prime}{ }_{5(0.25)}$ & 0.1364 & 0.1169 & 0.0975 & 0.0781 & 0.0586 & 0.0489 & 0.0457 & 0.0441 & 0.0431 \\
\hline$N^{* \prime}{ }_{5(0.05)}$ & 0.0801 & 0.0746 & 0.0690 & 0.0634 & 0.0579 & 0.0551 & 0.0542 & 0.0537 & 0.0535 \\
\hline$N^{\prime} 2^{5}+10+1$ & \multicolumn{9}{|l|}{0.0576} \\
\hline$N_{2}^{\prime}$ & 0.1128 & 0.0907 & 0.0687 & 0.0466 & 0.0245 & 0.0135 & 0.0098 & 0.0079 & 0.0068 \\
\hline$N_{6(0.25)}^{* \prime}$ & 0.0858 & 0.0694 & 0.0538 & 0.0382 & 0.0226 & 0.0148 & 0.0122 & 0.0109 & 0.0101 \\
\hline$N_{6(0.05)}^{* \prime}$ & 0.0489 & 0.0417 & 0.0345 & 0.0273 & 0.0200 & 0.0164 & 0.0152 & 0.0146 & 0.0143 \\
\hline$N^{\prime} 2^{6}+12+1$ & \multicolumn{9}{|l|}{0.0179} \\
\hline
\end{tabular}


Table 3. Values of $N^{\prime}{ }_{2 k}, N^{* \prime}{ }_{k(\alpha)}$ and $N^{\prime}{ }_{2}{ }^{k+2 k+1}$ when $\sigma^{2} / \beta^{* 2}=150$.

\begin{tabular}{|c|c|c|c|c|c|c|c|c|c|}
\hline$\sigma^{-2}($ Norm $)$ & 5 & 4 & 3 & 2 & 1 & $\frac{1}{2}$ & $\frac{1}{3}$ & $\frac{1}{4}$ & $-\frac{1}{5}$ \\
\hline$N^{\prime} 2^{3}$ & 0.3182 & 0.2877 & 0.2572 & 0.2266 & 0.1961 & 0.1808 & 0.1758 & 0.1732 & 0.1713 \\
\hline$N^{* \prime}{ }_{3(0.25)}$ & 0.2899 & 0.2820 & 0.2742 & 0.2664 & 0.2586 & 0.2547 & 0.2534 & 0.2527 & 0.2523 \\
\hline$N^{* \prime} 3(0.05)$ & 0.2809 & 0.2802 & 0.2797 & 0.2791 & 0.2785 & 0.2781 & 0.2781 & 0.2780 & 0.2780 \\
\hline$N^{\prime} 2^{3}+6+1$ & \multicolumn{9}{|l|}{0.2801} \\
\hline$N_{24}^{\prime}$ & 0.1493 & 0.1264 & 0.1034 & 0.0805 & 0.0575 & 0.0460 & 0.0422 & 0.0403 & 0.0391 \\
\hline$N^{* \prime}{ }_{24}(0.25)$ & 0.1085 & 0.1020 & 0.0954 & 0.0889 & 0.0823 & 0.0791 & 0.0780 & 0.0774 & 0.0771 \\
\hline$N^{* \prime}{ }_{24}^{4}(0.05)$ & 0.0957 & 0.0943 & 0.0929 & 0.0915 & 0.0901 & 0.0894 & 0.0892 & 0.0891 & 0.0890 \\
\hline$N^{\prime}{ }^{\prime}{ }^{4}+8+1$ & \multicolumn{9}{|l|}{0.0922} \\
\hline$N^{\prime} 2^{5}$ & 0.1662 & 0.1359 & 0.1055 & 0.0752 & 0.0448 & 0.0296 & 0.0246 & 0.0221 & 0.0205 \\
\hline$N^{* \prime}{ }_{25}{ }^{*}(0.25)$ & 0.0978 & 0.0866 & 0.0753 & 0.0641 & 0.0529 & 0.0473 & 0.0454 & 0.0445 & 0.0439 \\
\hline$N^{* \prime}{ }_{25}{ }^{5}(0.05)$ & 0.0680 & 0.0651 & 0.0622 & 0.0593 & 0.0564 & 0.0549 & 0.0545 & 0.0542 & 0.0541 \\
\hline$N^{\prime} 2^{5}+10+1$ & \multicolumn{9}{|l|}{0.0576} \\
\hline$N^{\prime}{ }^{6}{ }^{6}$ & 0.0760 & 0.0613 & 0.0466 & 0.0319 & 0.0171 & 0.0098 & 0.0073 & 0.0061 & 0.0054 \\
\hline$N_{6(0.25)}^{* \prime \prime}$ & 0.0515 & 0.0430 & 0.0345 & 0.0260 & 0.0175 & 0,0132 & 0.0118 & 0.0111 & 0.0106 \\
\hline$N_{6(0.05)}^{*^{\prime}}$ & 0.0303 & 0.0272 & 0.0240 & 0.0209 & 0.0177 & 0.0161 & 0.0156 & 0.0154 & 0.0152 \\
\hline$N^{\prime}{ }_{2}^{6}+12+1$ & \multicolumn{9}{|l|}{0.0179} \\
\hline
\end{tabular}

Table 4. Values of $N^{\prime}{ }_{2}{ }, N^{* \prime}{ }_{k(\alpha)}$ and $N^{\prime}{ }^{k}+2 k+1$ when $\sigma^{2} / \beta^{* 2}=200$.

\begin{tabular}{|c|c|c|c|c|c|c|c|c|c|}
\hline$\sigma^{-2}($ Norm $)$ & 5 & 4 & 3 & 2 & 1 & $\frac{1}{2}$ & $\frac{1}{3}$ & $\begin{array}{l}1 \\
4\end{array}$ & $\frac{1}{5}$ \\
\hline$N^{\prime}{ }^{3}$ & 0.2801 & 0.2572 & 0.2343 & 0.2114 & 0.1885 & 0.1770 & 0.1732 & 0.1713 & 0.1702 \\
\hline$N^{* \prime} 3(0.25)$ & 0.2801 & 0.2743 & 0.2685 & 0.2627 & 0.2568 & 0.2539 & 0.2530 & 0.2525 & 0.2522 \\
\hline$N^{* \prime}{ }_{3(0.05)}$ & 0.2801 & 0.2797 & 0.2792 & 0.2788 & 0.2783 & 0.2781 & 0.2780 & 0.2780 & 0.2780 \\
\hline$N^{\prime} 2^{3}+6+1$ & \multicolumn{9}{|l|}{0.2801} \\
\hline$N_{24}^{\prime}$ & 0.1206 & 0.1034 & 0.0862 & 0.0690 & 0.0518 & 0.0431 & 0.0403 & 0.0388 & 0.0380 \\
\hline$N^{* \prime}{ }_{24}^{4}(0.25)$ & 0.1001 & 0.0953 & 0.0906 & 0.0858 & 0.0811 & 0.0787 & 0.0779 & 0.0775 & 0.0773 \\
\hline$N^{*{ }^{1}}(0.05)$ & 0.0939 & 0.0929 & 0.0919 & 0.0909 & 0.0899 & 0.0894 & 0.0892 & 0.0891 & 0.0891 \\
\hline$N^{\prime} 2^{4}+8+1$ & \multicolumn{9}{|l|}{0.0922} \\
\hline$N^{\prime}{ }^{5}$ & 0.1283 & 0.1055 & 0.0828 & 0.0600 & 0.0372 & 0.0259 & 0.0221 & 0.0202 & 0.0190 \\
\hline$N^{* \prime}{ }_{25}(0.25)$ & 0.0816 & 0.0739 & 0.0662 & 0.0584 & 0.0507 & 0.0468 & 0.0455 & 0.0449 & 0.0445 \\
\hline$N_{25}^{* \prime}(0.05)$ & 0.0634 & 0.0616 & 0.0597 & 0.0578 & 0.0559 & 0.0550 & 0.0547 & 0.0545 & 0.0544 \\
\hline$N^{\prime}{ }^{5}+10+1$ & \multicolumn{9}{|l|}{0.0576} \\
\hline$N_{2}^{\prime}{ }^{6}$ & 0.0576 & 0.0466 & 0.0355 & 0.0245 & 0.0135 & 0.0079 & 0.0061 & 0.0052 & 0.0046 \\
\hline$N^{* \prime}{ }^{* 6}(0.25)$ & 0.0377 & 0.0322 & 0.0267 & 0.0212 & 0.0157 & 0.0129 & 0.0120 & 0.0115 & 0.0113 \\
\hline$N_{26}^{* \prime}(0.05)$ & 0.0243 & 0.0225 & 0.0208 & 0.0190 & 0.0172 & 0.0163 & 0.0160 & 0.0158 & 0.0157 \\
\hline$N_{26}^{\prime}+12+1$ & \multicolumn{9}{|l|}{0.0179} \\
\hline
\end{tabular}




\section{References}

[1] T. A. BANCROFT: On biases in estimation due to the use of preliminary tests of significance. Ann. Math. Stst., 15 (1944), 190-204.

[2] G. E. P. Box and J.S. Honter: Multi-factor experimental designs for exploring response surfaces. Ann. Math. Stat., 28 (1957), 195-241.

[3] G. E. P. Box and K. B WILson: On the experimental attainment of optimum conditions. J. Roy. Stat. Soc., Ser. B, 13 (1951), 1-45.

[4] T. Kitagawa: Successive process of statistical inferences. Mem. Fac. Sci. Kyushu Univ., Ser. A, 15 (1950), 139-180.

[5] T. Kitagawa: Tables of the Poisson distribution. Japan, 1951.

[6] T. KitagaWa: Successive process of statistical inferences applied to linear regression analysis and its specialisations to response surface. Bull. Math. Stats., 9 (1959),

[7] A. E. PAULL: On a preliminary test for pooling mean squares in the analysis of variance. Ann. Math. Stat., 21 (1950), 539-556.

[8] K. Pearson: Tables of the incomplete beta-function. Cambridge, 1934.

Editorial: This paper is the last publication by Miss Michie Shirafuji who has died on February 6, 1959 in Fukuoka. 